\title{
Pharmacokinetic/pharmacodynamic relationships of FTY720 in kidney transplant recipients
}

S.I. Park ${ }^{1}$, C.R. Felipe ${ }^{1}$, P.G. Machado ${ }^{1}$, R. Garcia ${ }^{1}$, A. Skerjanec ${ }^{2}$, R. Schmouder ${ }^{2}$, H. Tedesco-Silva Jr. ${ }^{1}$ and J.O. Medina-Pestana ${ }^{1}$
1Divisão de Nefrologia, Hospital do Rim e Hipertensão, Escola Paulista de Medicina, Universidade Federal de São Paulo, São Paulo, SP, Brasil

${ }^{2}$ Novartis Pharmaceuticals, East Hanover, NJ, USA

\section{Correspondence \\ H. Tedesco-Silva Jr. \\ Divisão de Nefrologia \\ Hospital do Rim e Hipertensão \\ EPM, UNIFESP \\ Rua Borges Lagoa, 960, 119 andar \\ 04038-002 São Paulo, SP \\ Brasil \\ Fax: + 55-11-5087-8008 \\ E-mail: heliotedesco@hrim.com.br or parksung@hotmail.com \\ Part of the data were presented at the American Transplant Congress, May 30-June 4, 2003, Washington, DC, USA.}

Publication supported by FAPESP.

Received March 22, 2004 Accepted February 3, 2005

\begin{abstract}
FTY720 is a new and effective immunosuppressive agent, which produces peripheral blood lymphopenia through a lymphocyte homing effect. We investigated the relationship between the dose of FTY720 or blood concentration (pharmacokinetics, PK) and peripheral lymphopenia (pharmacodynamics, PD) in 23 kidney transplant recipients randomized to receive FTY720 (0.25-2.5 mg/day) or mofetil mycophenolate ( $2 \mathrm{mg}$ /day) in combination with cyclosporine and steroids. FTY720 dose, blood concentrations and lymphocyte counts were determined weekly before and 4 to 12 weeks after transplantation. The effect of PD was calculated as the absolute lymphocyte count or its reductions. PK/PD modeling was used to find the best-fit model. Mean FTY720 concentrations were $0.36 \pm$ $0.05(0.25 \mathrm{mg}), 0.73 \pm 0.12(0.5 \mathrm{mg}), 3.26 \pm 0.51(1 \mathrm{mg})$, and $7.15 \pm 1.41$ $\mathrm{ng} / \mathrm{ml}(2.5 \mathrm{mg})$ between 4 and 12 weeks after transplantation. FTY720 PK was linear with dose $\left(r^{2}=0.98\right)$ and showed low inter- and intraindividual variability. FTY720 produced a dose-dependent increase in mean percent reduction of peripheral lymphocyte counts (38 vs 42 vs 56 vs 77, $\mathrm{P}<0.01$, respectively). The simple $\mathrm{E}_{\max }$ model $\left[\mathrm{E}=\left(\mathrm{E}_{\max } * \mathrm{C}\right) /\left(\mathrm{C}+\mathrm{EC}_{50}\right)\right]$ was the best-fit PK/PD modeling for FTY720 dose $\left(\mathrm{E}_{\max }=87.8 \pm 5.3 \%\right.$ and $\left.\mathrm{ED}_{50}=0.48 \pm 0.08 \mathrm{mg}, \mathrm{r}^{2}=0.94\right)$ or concentration $\left(\mathrm{E}_{\max }=78.3 \pm 2.9 \%\right.$ and $\mathrm{EC}_{50}=0.59 \pm 0.09 \mathrm{ng} / \mathrm{ml}$, $\left.\mathrm{r}^{2}=0.89\right) v s$ effect (\% reduction in peripheral lymphocytes). FTY720 $\mathrm{PK} / \mathrm{PD}$ is dose dependent and follows an $\mathrm{E}_{\max }$ model $\left(\mathrm{EC}_{50}=0.5 \mathrm{mg}\right.$ or $0.6 \mathrm{ng} / \mathrm{ml})$. Using lymphopenia as an FTY720 PD surrogate marker, high \% reductions ( 80\%) in peripheral lymphocytes are required to achieve best efficacy to prevent acute allograft rejection.
\end{abstract}

\section{Introduction}

FTY720 (2-amino-2-[2-(4-octylphenyl) ethyl] propane-1,3 diol hydrochloride) is a novel immunomodulator agent developed by a chemical modification of myriocine (1-3),
Key words

- FTY720

- Lymphopenia

- Pharmacokinetics

- Pharmacodynamics

- Immunosuppression

- Renal transplants 
synergistic interactions with cyclosporine, tacrolimus, sirolimus, or everolimus (6-14). FTY720 is currently undergoing phase III clinical trials in kidney transplant recipients after FTY720 doses and combination therapy were identified in phase II studies.

FTY720 produces peripheral lymphopenia in both animals and humans as a consequence of altered T-cell trafficking and redirection to secondary lymphatic organs (15, 16). At therapeutic concentrations, FTY720 does not interfere with cytokine synthesis or binding, does not inhibit cell proliferation and does not cause cell death by apoptosis $(17,18)$. Lymphopenia is fully reversible upon drug discontinuation (19). FTY720 needs to be phosphorylated by sphingosine phosphatase and the phosphorylated form (FTY720-P) appears to mediate its biological effect through the binding to sphingosine-1-phosphate, Gprotein-coupled receptors expressed in the membrane of lymphocytes and endothelial cell of lymph nodes (20-22). The proposed molecular mechanism of action is that lymphocyte homing is altered by FTY720 as a sphingosine-1-phosphate agonist targeting G-protein-coupled receptors expressed in the membrane of lymphocytes and endothelial cell of lymph nodes (23). This binding results in drug-receptor internalization, increasing intrinsic lymphocyte mobility and chemotactic response, accelerating lymphocyte homing, and trapping in secondary lymphoid tissues $(21,24)$.

FTY720 has unique pharmacokinetic properties. Its absorption is slow, reaching peak concentrations 8 to $36 \mathrm{~h}$ after oral administration (19). FTY720 has a large volume of distribution (1116-1737 L) and a clearance of about 123 to $383 \mathrm{ml} / \mathrm{min}$, resulting in a long elimination half-life of about 108 $\mathrm{h}$ (4.5 days) (19). Since it is given once a day, presumably it takes about 4 weeks to reach steady-state concentration, with 11- to 19fold accumulation in tissues compared to first dose administration (19).

Pharmacokinetic and pharmacodynamic
(PK/PD) modeling has been used increasingly in clinical pharmacology and drug development, not only to speed up the development process but, more importantly, to determine the optimal dosage of new drugs, which will deliver an appropriate effect. The relationship between FTY720 pharmacokinetics and pharmacodynamics has been studied in various experimental transplant models (13). The aim of the present study was to determine the relationship among FTY720 dose, blood concentration and surrogate biological effect in the peripheral blood compartment (lymphopenia). This information will help to choose and possibly individualize drug dose regimens, which will result in best efficacy/toxicity relationships.

\section{Material and Methods}

\section{Population}

Between June 1st and September 30, 2000, 23 kidney transplant recipients were enrolled at our center as part of an international multicenter prospective, open-label, randomized, dose-finding, and exploratory trial, which included 208 patients. The primary objective was to evaluate the safety, tolerability and preliminary efficacy of increasing doses of FTY720 versus mofetil mycophenolate (MMF, CellCept ${ }^{\circledR}$, Roche Laboratories, New Jersey, NJ, USA) in combination with a cyclosporine microemulsion and prednisone.

The local Medical Ethics Committee approved the protocol and the study was performed in accordance with the Declaration of Helsinki and US Food and Drug Administration guidelines for good clinical practice. All patients signed an informed consent term after being informed of the details of this study, and were enrolled according to study-specific inclusion and exclusion criteria. The present report describes a retrospective analysis of data obtained from 23 patients enrolled at our transplant center during the clinical trial. 


\section{Study design}

The study involved 24 weeks of observation divided into 12 weeks of treatment and 12 weeks of follow-up. Patients were randomized $24 \mathrm{~h}$ after renal transplantation to one of four FTY720 groups $(0.25 \mathrm{mg}(\mathrm{N}=$ 4), $0.5 \mathrm{mg}(\mathrm{N}=4), 1 \mathrm{mg}(\mathrm{N}=5), 2.5 \mathrm{mg}(\mathrm{N}$ $=5)$ ) or to one MMF group $(\mathrm{N}=5)$. To be enrolled in the study patients had to be between 18 and 65 years old and to be receiving their first living or cadaver donor allografts. All patients received initial doses of cyclosporine of 8 to $10 \mathrm{mg} \mathrm{kg}^{-1}$ day $^{-1}$ twice a day adjusted to achieve therapeutic concentrations of $200-400 \mathrm{ng} / \mathrm{ml}$ during the first month, $150-250 \mathrm{ng} / \mathrm{ml}$ during the second and third months, and 100-200 ng/ml thereafter. Prednisone was introduced two days after the transplant, with initial doses of $0.5 \mathrm{mg} \mathrm{kg}^{-1}$ day $^{-1}$ followed by a gradual reduction to $0.2 \mathrm{mg} \mathrm{kg}^{-1} \mathrm{day}^{-1}$ at 12 weeks post-transplant. Within $24 \mathrm{~h}$ after transplant surgery, a loading dose of 1, 2, 4, and $4 \mathrm{mg}$ was administered to patients randomized to receive fixed maintenance doses of 0.25 , $0.5,1$, or $2.5 \mathrm{mg} /$ day of FTY720. Patients randomized to the MMF group received $2 \mathrm{~g}$ twice a day. FTY720 treatment lasted 12 weeks with the patients being converted to azathioprine, $2 \mathrm{mg} \mathrm{kg}^{-1} \mathrm{day}^{-1}$, or MMF, $2 \mathrm{~g} /$ day thereafter at the discretion of the investigator. Drug doses were also adjusted for safety and tolerance.

\section{Pharmacokinetics/pharmacodynamics}

There were 14 study visits, namely pretransplant and at 1, 2, 4 to 12 weeks during the treatment phase and 16, 20 and 24 weeks during the follow-up phase. On the occasion of each study visit, blood samples were obtained to measure blood concentrations of cyclosporine and FTY720 and to perform lymphocyte counts.

Pharmacokinetic analysis. Whole blood cyclosporine concentrations were measured daily during the first week and doses were adjusted until therapeutic concentrations (>200 ng/ml) were achieved. Subsequent monitoring was performed twice a week during the first month, once a week during the second month, and every other week thereafter. Whole blood cyclosporine concentrations were measured by a fluorescence polarization immunoassay (Abbott Laboratories, Chicago, IL, USA) according to the manufacturer instructions. Since FTY720 has a long terminal half-live of about $100 \mathrm{~h}$, steady-state concentrations are achieved only after 4 weeks of treatment. Therefore, blood samples were collected to measure whole blood FTY720 concentrations between 4 and 12 weeks. Blood concentrations of FTY720 were determined using a validated HPLC/mass spectrometry/ mass spectrometry method from Novartis (East Hanover, NJ, USA).

Pharmacodynamics study. The surrogate marker of the pharmacodynamic effect of FTY720 used in this study was the peripheral lymphocyte count in the blood compartment. Lymphocyte counts were performed on the occasion of each study visit (pretransplant and 1, 2, 4, 5, 6, 7, 8, 9, 10, 11, 12, 16,20 , and 24 weeks after transplant) in all 5 groups using the CELL-DYN 3200 automatic counter (Abbott Park, Chicago, IL, USA) whose methods of determinations are based on flow cytometry. Since MMF has no effect on peripheral lymphocytes (25), lymphocyte counts obtained from patients receiving MMF were used to calculate the basal pharmacodynamic effect when FTY720 doses or exposures were equal to zero. The pharmacodynamic effect of FTY720 was determined either as absolute reduction in peripheral lymphocyte counts or as percent reduction compared to the lymphocyte count obtained pre-transplant and before the administration of the first dose of FTY720 or MMF.

PK/PD correlations. Peripheral lymphocyte counts were correlated with FTY720 
doses and blood concentrations. PK/PD modeling was used to find the best-fit model of the correlation between absolute or percent reduction in peripheral lymphocyte count and increasing doses or blood concentrations of FTY720. For these analysis we used the scientific software WinNonlin (SCI Software) to find the best fit PK/PD model that explains the relationship between FTY720 dose or blood concentration and the chosen pharmacodynamic surrogate marker.

\section{Statistical analysis}

Demographic characteristics were analyzed by analysis of variance (ANOVA) for continuous variables and by the chi-square test for categorical variables. Summary statistics were expressed as means $\pm \mathrm{SD}$, or frequencies or median and range, respectively. The observed and calculated PK/PD parameters were presented as means $\pm \mathrm{SD}$ and as dose/response and exposure/response relationships. Linear regression analyses were used to correlate FTY720 dose and blood concentration. Nonlinear regressions were used to correlate the dose and drug concentration in blood with biological response. All statistical analyses were performed using the SPSS 7.5 software (SPSS Inc., Chicago, IL, USA), with the level of significance set at $P$ $<0.05$.

\section{Results}

\section{Demographics and baseline characteristics}

Demographic characteristics of the allograft recipients were similar across treatment groups (Table 1). Mean age was 40.1 \pm 11.0 years, weight $62.2 \pm 8.8 \mathrm{~kg}$, and mean body mass index was $22.8 \pm 2.6 \mathrm{~kg} / \mathrm{m}^{2}$. Most patients were males $(61 \%)$ and white $(61 \%)$, with $4(17 \%)$ blacks and $5(22 \%)$ with various degrees of miscegenation. The primary etiology leading to renal failure was chronic glomerulonephritis (22\%), followed by diabetes $(17 \%)$ and nephrosclerosis (13\%). Mean number of HLA mismatches was $2.9 \pm 0.5$ and $100 \%$ showed no preformed anti-HLA antibodies. All recipients tested negative for hepatitis $\mathrm{B}$ and $\mathrm{C}$ viruses and $50 \%$ of the recipients were positive for cytomegalovirus IgG. No significant differences in demographic characteristics were observed between the 5 groups.

\section{Pharmacokinetics}

Cyclosporine doses and whole blood concentrations did not differ statistically between the 5 groups during the 24 weeks of treatment. Mean doses/weight were $9.7 \pm$ 2.5 in the first week, $6.5 \pm 1.9$ at week $4,4.7$ \pm 1.5 at week 12 , and $3.8 \pm 1.5 \mathrm{mg} \mathrm{kg}^{-1}$ day $^{-1}$ at week 24 , with no significant differences between groups (Figure 1A). Corresponding mean whole blood cyclosporine concentrations were $271.0 \pm 104.1,351.1 \pm$ $121.4,213.5 \pm 71.3$, and $149.1 \pm 72.7 \mathrm{ng} / \mathrm{ml}$, respectively (Figure 1B). Also dose-normalized cyclosporine concentrations did not differ significantly between the 5 groups, ranging from $0.50 \pm 0.35$ at week 1 to $0.63 \pm 0.30$ $\mathrm{ng} \mathrm{ml}^{-1} \mathrm{mg}^{-1}$ at week 24 . Therapeutic concentrations of cyclosporine were achieved in all groups during the study period. Mean prednisone doses were $30.4 \pm 3.7$ (week 1), $27.3 \pm 3.7$ (week 4), $13.3 \pm 3.7$ (week 12), and $9.9 \pm 0.5 \mathrm{mg} /$ day (week 24), with no significant differences between the 5 groups.

Mean FTY720 blood concentrations according to dose level and study visit are shown in Figure 2. There were no significant differences in mean blood concentration between study visits (General Linear Model for repeated measurements) within each dose level, confirming that at week 4 steady-state FTY720 concentrations had been achieved (Table 2 ). Interindividual variability (\% coefficients of variation, $\% \mathrm{CV}$ ) increased from 24 to $41 \%$ with increasing doses of FTY720 $(\mathrm{P}=0.05)$ but no significant differences were observed between study visits. Aver- 
Table 1. Demographic characteristics of the transplant population.

\begin{tabular}{|c|c|c|c|c|c|c|}
\hline & Total & $\begin{array}{l}\text { FTY720 } \\
(0.25 \mathrm{mg})\end{array}$ & $\begin{array}{l}\text { FTY720 } \\
\text { (0.5 mg) }\end{array}$ & $\begin{array}{c}\text { FTY720 } \\
(1 \mathrm{mg})\end{array}$ & $\begin{array}{l}\text { FTY720 } \\
(2.5 \mathrm{mg})\end{array}$ & $\begin{array}{l}\text { MMF } \\
(2 \mathrm{~g})\end{array}$ \\
\hline$N$ & 23 & 4 & 4 & 5 & 5 & 5 \\
\hline Age (years) & $\begin{array}{c}40.1 \pm 11.0 \\
(23.0-59.0)\end{array}$ & $\begin{array}{l}45.5 \pm 5.0 \\
(40.0-52.0)\end{array}$ & $\begin{array}{l}38.3 \pm 14.0 \\
(23.0-52.0)\end{array}$ & $\begin{array}{c}40.4 \pm 14.3 \\
(24.0-59.0)\end{array}$ & $\begin{array}{c}38.6 \pm 11.8 \\
(24.0-57.0)\end{array}$ & $\begin{array}{c}38.6 \pm 10.7 \\
(25.0-52.0)\end{array}$ \\
\hline Gender (male/female) & $14 / 9$ & $3 / 1$ & $3 / 1$ & $0 / 5$ & $4 / 1$ & $4 / 1$ \\
\hline Weight $(\mathrm{kg})$ & $\begin{array}{l}62.2 \pm 8.8 \\
(47.4-83.6)\end{array}$ & $\begin{array}{l}68.6 \pm 10.1 \\
(62.3-83.6)\end{array}$ & $\begin{array}{l}60.4 \pm 5.6 \\
(53.1-65.9)\end{array}$ & $\begin{array}{c}55.6 \pm 7.5 \\
(47.4-61.8)\end{array}$ & $\begin{array}{c}61.8 \pm 10.4 \\
(53.8-79.7)\end{array}$ & $\begin{array}{c}65.4 \pm 7.2 \\
(57.6-76.0)\end{array}$ \\
\hline $\mathrm{BMI}\left(\mathrm{kg} / \mathrm{m}^{2}\right)$ & $\begin{array}{l}22.8 \pm 2.6 \\
(19.2-27.0)\end{array}$ & $\begin{array}{l}24.7 \pm 2.2 \\
(22.3-27.0)\end{array}$ & $\begin{array}{l}22.1 \pm 1.7 \\
(20.5-24.2)\end{array}$ & $\begin{array}{l}23.9 \pm 3.8 \\
(19.2-27.0)\end{array}$ & $\begin{array}{l}21.5 \pm 2.0 \\
(20.1-24.9)\end{array}$ & $\begin{array}{c}22.0 \pm 2.5 \\
(19.7-26.0)\end{array}$ \\
\hline Ethnicity (white/black/mulatto/others) & $14 / 4 / 2 / 3$ & $3 / 0 / 0 / 1$ & $4 / 0 / 0 / 0$ & $2 / 1 / 1 / 1$ & $3 / 1 / 0 / 1$ & $2 / 2 / 1 / 0$ \\
\hline HLA mismatches & $2.9 \pm 0.5$ & $3.3 \pm 0.5$ & $3.0 \pm 0.0$ & $2.6 \pm 0.5$ & $2.8 \pm 0.4$ & $2.9 \pm 0.5$ \\
\hline \multicolumn{7}{|l|}{ Cause of ESRD } \\
\hline Chronic glomerulonephritis & $5(21.7 \%)$ & 2 & 1 & 0 & 1 & 1 \\
\hline Nephrosclerosis & $3(13.0 \%)$ & 0 & 0 & 1 & 1 & 1 \\
\hline Polycystic kidney disease & $2(8.9 \%)$ & 0 & 0 & 0 & 1 & 1 \\
\hline Diabetic nephropathy & $4(17.4 \%)$ & 1 & 1 & 1 & 0 & 1 \\
\hline Unknown & $9(39.1 \%)$ & 1 & 2 & 3 & 2 & 1 \\
\hline Time on dialysis (months) & $\begin{array}{l}17.7 \pm 10.2 \\
(2.0-36.0)\end{array}$ & $\begin{array}{l}22.3 \pm 12.9 \\
(6.0-36.0)\end{array}$ & $\begin{array}{l}18.5 \pm 4.9 \\
(12.0-24.0)\end{array}$ & $\begin{array}{c}20.0 \pm 13.6 \\
(2.0-36.0)\end{array}$ & $\begin{array}{c}16.2 \pm 11.3 \\
(7.0-36.0)\end{array}$ & $\begin{array}{r}12.6 \pm 6.8 \\
(5.0-23.0)\end{array}$ \\
\hline Panel reactive antibody $<5 / \geq 5(\%)$ & $23 / 0$ & $4 / 0$ & $4 / 0$ & $5 / 0$ & $5 / 0$ & $5 / 0$ \\
\hline \multicolumn{7}{|l|}{ Viral status } \\
\hline Anti-HCV positive (lgG) & 0 & 0 & 0 & 0 & 0 & 0 \\
\hline HbsAg positive & 0 & 0 & 0 & 0 & 0 & 0 \\
\hline CMV positive (lgG) & 12 & 3 & 1 & 2 & 3 & 3 \\
\hline
\end{tabular}

Data are reported as means \pm SD (range). MMF = mofetil mycophenolate; $B M I=$ body mass index; HLA = human leukocyte antigen; ESRD = end-stage renal disease; $\mathrm{HCV}=$ hepatitis $\mathrm{C}$ virus; $\mathrm{CMV}=$ cytomegalovirus. Panel reactive antibody between donor and recipients greater or less than $5 \%$.
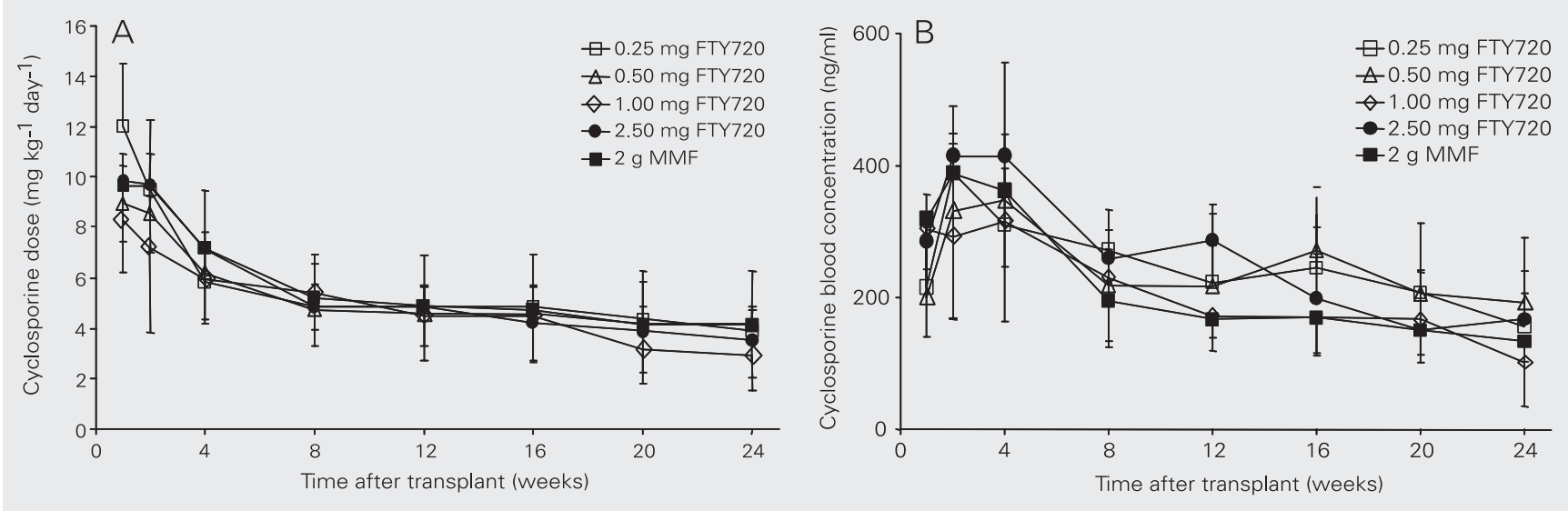

Figure 1. Time course of mean cyclosporine doses (A) and blood concentrations (B). There were no significant differences for mean cyclosporine doses or blood concentrations among study groups at each visit (independent Student $t$-test). MMF = mofetil mycophenolate. 


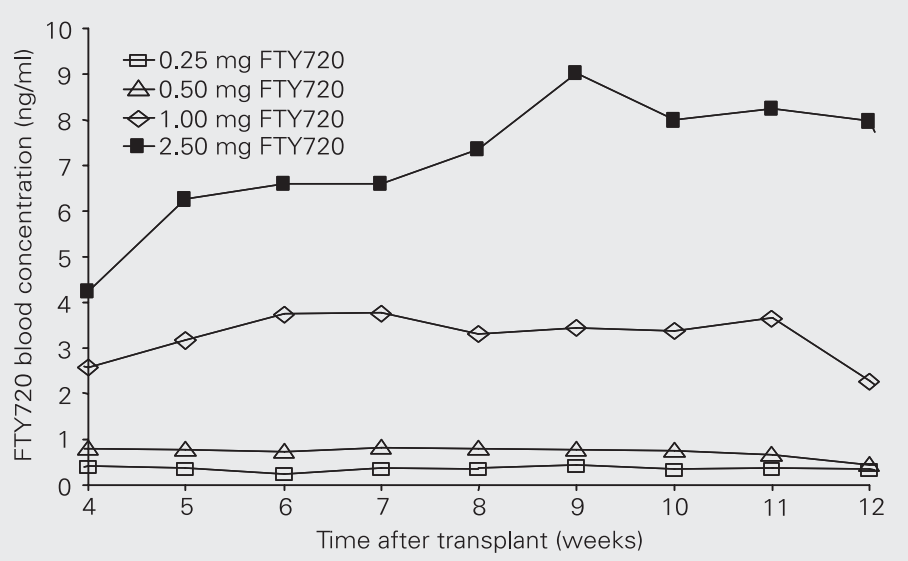

Figure 2. Time course of mean FTY720 blood concentrations between 4 and 12 weeks post-transplant. $\mathrm{P}<0.01$ for 1.0 and $2.5 \mathrm{mg}$ FTY720 compared to 0.25 and $0.5 \mathrm{mg}$ FTY720 during administration time (ANOVA). age intra-individual variability $(\% \mathrm{CV})$ ranged from 25 to 28 and was not dose dependent. In the 10 -fold dose range studied in this trial ( 0.25 to $2.5 \mathrm{mg}$ /day), FTY720 pharmacokinetics was linear with dose $\left(r^{2}=0.679\right.$ and 0.982), as can be observed in Figure 3.

\section{Pharmacodynamics}

The time course of the FTY720-induced reduction of peripheral lymphocyte count is shown in Figure 4. There were no significant differences in pre-transplant values between groups and lymphopenia was not observed in the MMF group throughout the study. The

Table 2. FTY720 blood concentrations and percent coefficient of variation (\%CV) between weeks 4 and 12 according to FTY720 dose level.

\begin{tabular}{lcccc}
\hline Visit (week) & $\begin{array}{c}\text { FTY720 }(0.25 \mathrm{mg}) \\
\mathrm{N}=4\end{array}$ & $\begin{array}{c}\text { FTY720 }(0.50 \mathrm{mg}) \\
\mathrm{N}=4\end{array}$ & $\begin{array}{c}\text { FTY720 (1.00 mg) } \\
\mathrm{N}=5\end{array}$ & $\begin{array}{c}\text { FTY720 (2.50 mg) } \\
\mathrm{N}=5\end{array}$ \\
\hline $4^{\mathrm{a}}$ & $0.41 \pm 0.13(32)$ & $0.81 \pm 0.13(16)$ & $2.59 \pm 1.08(42)$ & $4.26 \pm 1.65(39)$ \\
$5^{\mathrm{a}}$ & $0.37 \pm 0.12(32)$ & $0.78 \pm 0.16(21)$ & $3.18 \pm 1.35(42)$ & $6.25 \pm 2.80(45)$ \\
$6^{\mathrm{a}}$ & $0.26 \pm 0.05(14)$ & $0.72 \pm 0.26(26)$ & $3.74 \pm 2.51(67)$ & $6.60 \pm 1.82(29)$ \\
$7^{a}$ & $0.36 \pm 0.16(44)$ & $0.81 \pm 0.25(31)$ & $3.77 \pm 1.39(37)$ & $6.61 \pm 1.81(27)$ \\
$8^{a}$ & $0.37 \pm 0.01(3)$ & $0.80 \pm 0.36(45)$ & $3.31 \pm 1.05(32)$ & $7.36 \pm 2.58(36)$ \\
$9^{a}$ & $0.43 \pm 0.11(26)$ & $0.79 \pm 0.25(32)$ & $3.44 \pm 1.53(44)$ & $9.02 \pm 2.82(32)$ \\
$10^{a}$ & $0.33 \pm 0.07(21)$ & $0.76 \pm 0.31(41)$ & $3.37 \pm 0.66(20)$ & $8.00 \pm 2.56(32)$ \\
$11^{a}$ & $0.37 \pm 0.09(24)$ & $0.66 \pm 0.27(41)$ & $3.65 \pm 1.09(30)$ & $8.24 \pm 4.17(51)$ \\
$12^{a}$ & $0.35 \pm 0.08(23)$ & $0.44 \pm 0.30(68)$ & $2.28 \pm 1.22(54)$ & $7.97 \pm 4.05(51)$ \\
Total a & $0.36 \pm 0.05(24)$ & $0.73 \pm 0.12(36)$ & $3.26 \pm 0.51(41)$ & $7.15 \pm 1.41(38)$ \\
Average interindividual \%CVb,c (range) & $24 \pm 12(14-44)$ & $36 \pm 15(16-68)$ & $41 \pm 14(20-67)$ & $38 \pm 9(27-51)$ \\
Average intra-individual \%CVd (range) & $25 \pm 14(7-40)$ & $26 \pm 16(12-42)$ & $25 \pm 8(17-35)$ & $28 \pm 13(14-49)$
\end{tabular}

Data are reported as means $\pm \mathrm{SD}$ in $\mathrm{ng} / \mathrm{ml}$ and \% CV within parentheses.

aFTY720 blood level, $\mathrm{P}<0.05$ between groups (ANOVA); binterindividual \%CV according to FTY720 dose, $\mathrm{P}=0.05$ (ANOVA); cinterindividual $\% \mathrm{CV}$ according to visit, $\mathrm{P}>0.05$ (general linear model for repeated measures); dintra-individual \% CV, P $>0.05$ between groups (ANOVA).
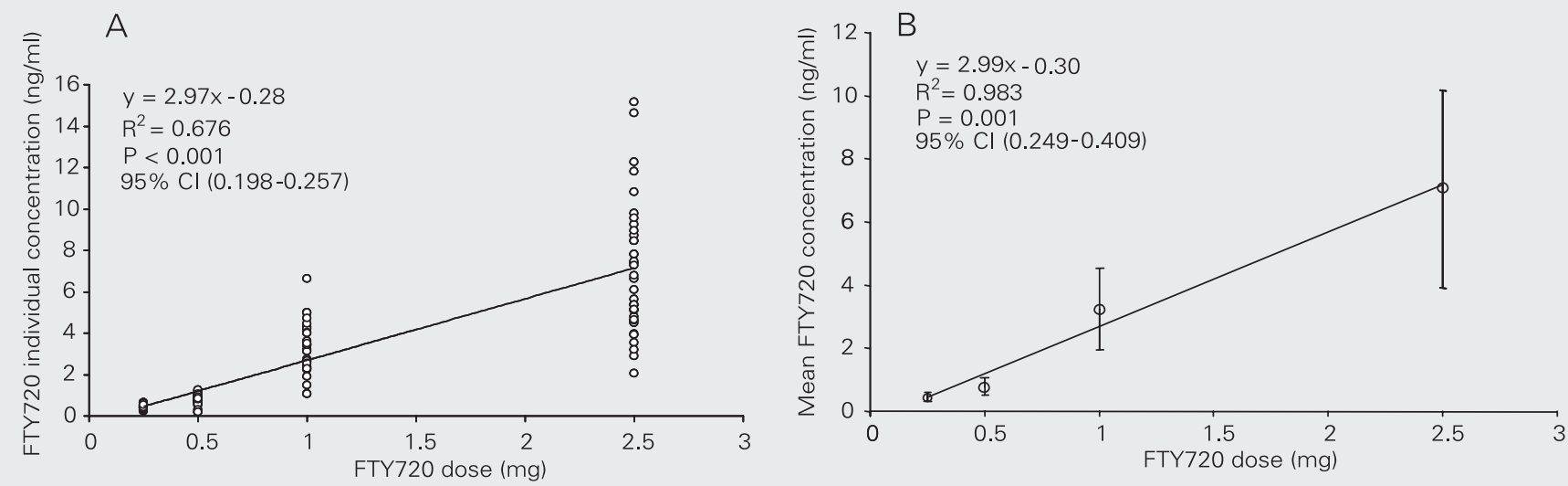

Figure 3. Linear regression analysis correlating individual (A) or mean (B) FTY720 blood concentrations and dose levels. 
reduction in the number of lymphocytes was observed as early as at week 1 , reached its peak at about week 4 , and was fully reversed 4 to 8 weeks after treatment interruption. The extent of lymphopenia and the time to return to baseline values appear to depend on the magnitude of the FTY720 dose. Mean percent reduction in lymphocyte counts after week 4 was dose dependent $(37.8 \pm 19.2$ $(0.25 \mathrm{mg})$ vs $42.4 \pm 19.9(0.50 \mathrm{mg})$ vs 56.0 $\pm 17.8(1.00 \mathrm{mg})$ vs $76.5 \pm 10.8 \%(2.50 \mathrm{mg})$, $\mathrm{P}<0.01)$. Mean lymphocyte counts varied from 624 to $1320(0.25 \mathrm{mg}), 675$ to 1085 (0.5 mg), 567 to $853(1 \mathrm{mg})$, and 327 to $696 /$ $\mathrm{mm}^{3}$ (2.5 mg; Table 3). Inter- (42-55\%) and intra-individual variability (25-55\%) ranges

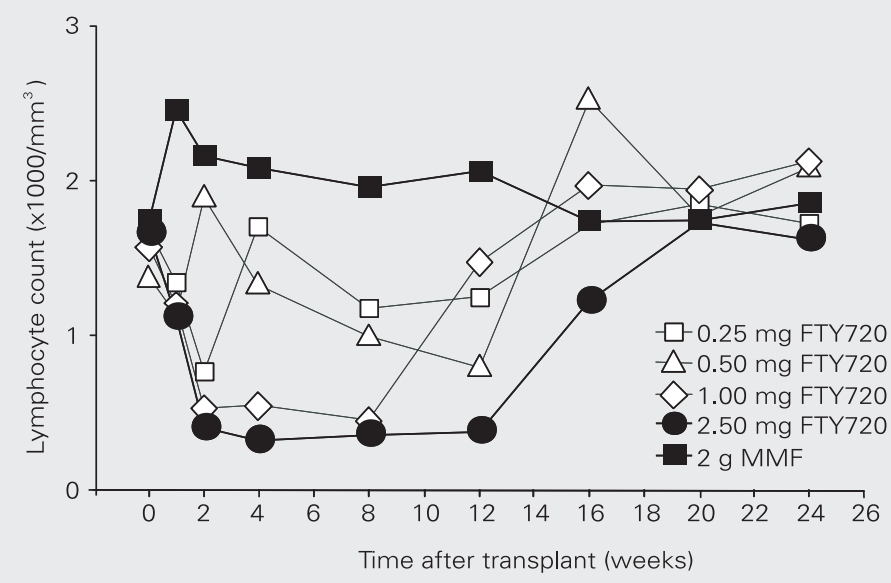

Figure 4. Time course of FTY720-induced lymphopenia during the 12-week treatment phase and return to baseline values upon drug discontinuation. MMF = mofetil mycophenolate.

Table 3. Mean peripheral blood lymphocyte counts and percent lymphocyte reduction between weeks 4 and 12 in the 5 groups of patients.

\begin{tabular}{|c|c|c|c|c|c|c|c|c|c|c|}
\hline \multirow{2}{*}{ Visit (week) } & \multicolumn{2}{|c|}{$\begin{array}{l}\mathrm{MMF}(2 \mathrm{~g}) \\
(\mathrm{N}=5)\end{array}$} & \multicolumn{2}{|c|}{$\begin{array}{c}\text { FTY720 (0.25 mg) } \\
(\mathrm{N}=4)\end{array}$} & \multicolumn{2}{|c|}{$\begin{array}{l}\text { FTY720 (0.5 mg) } \\
(\mathrm{N}=4)\end{array}$} & \multicolumn{2}{|c|}{$\begin{array}{l}\text { FTY720 (1 mg) } \\
\quad(\mathrm{N}=5)\end{array}$} & \multicolumn{2}{|c|}{$\begin{array}{c}\text { FTY720 (2.5 mg) } \\
(\mathrm{N}=5)\end{array}$} \\
\hline & $\begin{array}{l}\text { Lymphocytes } \\
\qquad\left(\mathrm{N} / \mathrm{mm}^{3}\right)\end{array}$ & $\begin{array}{c}\% \\
\text { Reduction }\end{array}$ & $\begin{array}{l}\text { Lymphocytes } \\
\qquad\left(\mathrm{N} / \mathrm{mm}^{3}\right)\end{array}$ & $\begin{array}{c}\% \\
\text { Reduction }\end{array}$ & $\begin{array}{l}\text { Lymphocytes } \\
\qquad\left(\mathrm{N} / \mathrm{mm}^{3}\right)\end{array}$ & $\begin{array}{c}\% \\
\text { Reduction }\end{array}$ & $\begin{array}{l}\text { Lymphocytes } \\
\left(\mathrm{N} / \mathrm{mm}^{3}\right)\end{array}$ & $\begin{array}{c}\% \\
\text { Reduction }\end{array}$ & $\begin{array}{l}\text { Lymphocytes } \\
\qquad\left(\mathrm{N} / \mathrm{mm}^{3}\right)\end{array}$ & $\begin{array}{c}\% \\
\text { Reduction }\end{array}$ \\
\hline \multicolumn{11}{|l|}{ Baseline } \\
\hline 0 & $1746(35)$ & - & $1677(21)$ & - & $1372(26)$ & - & 1567 (38) & - & 1657 (29) & - \\
\hline \multicolumn{11}{|c|}{ FTY720 treatment } \\
\hline $4^{a}$ & 2488 (8) & - & 872 (44) & $39.3(49)$ & $675(45)$ & $58.2(3)$ & $567(49)$ & $65.1(32)$ & $511(53)$ & $79.6(6)$ \\
\hline $5^{a}$ & $2059(45)$ & - & $624(59)$ & $46.6(66)$ & $805(69)$ & $52.6(4)$ & $674(61)$ & $59.0(43)$ & $459(68)$ & $82.6(12)$ \\
\hline $6^{a}$ & 2217 (34) & - & $1559(61)$ & $39.2(23)$ & $852(30)$ & $46.9(48)$ & $953(33)$ & $43.6(47)$ & $327(21)$ & $79.5(11)$ \\
\hline $7^{a}$ & $2344(28)$ & - & $900(75)$ & $43.8(49)$ & $890(35)$ & $44.1(61)$ & $588(50)$ & $62.9(34)$ & $663(33)$ & $69.9(10)$ \\
\hline $8^{a}$ & $2556(26)$ & - & 1067 (81) & $38.5(32)$ & $981(75)$ & $45.9(62)$ & $853(41)$ & $52.6(8)$ & $562(60)$ & $75.5(19)$ \\
\hline $9^{a}$ & $2518(25)$ & - & 1023 (98) & $49.2(40)$ & $837(35)$ & $46.8(41)$ & $845(46)$ & $48.8(45)$ & $645(88)$ & $78.8(12)$ \\
\hline $10^{a}$ & 2209 (27) & - & $1284(12)$ & $19.5(94)$ & $1085(28)$ & $23.4(84)$ & $800(42)$ & $52.3(33)$ & $696(70)$ & $74.0(13)$ \\
\hline $11^{a}$ & $2474(24)$ & - & $1320(20)$ & $15.1(42)$ & $934(36)$ & $45.8(42)$ & $724(17)$ & $53.4(45)$ & $545(52)$ & $76.9(11)$ \\
\hline $12^{a}$ & 2472 (46) & - & $924(12)$ & $26.1(4)$ & $1271(45)$ & $18.6(44)$ & $650(41)$ & $62.6(14)$ & $623(53)$ & $70.9(29)$ \\
\hline $4-12^{a}$ & 2364 (28) & - & $823(42)$ & $37.8(56)$ & 906 (49) & $42.4(47)$ & 731 (41) & $56.0(32)$ & 429 (37) & 76.5 (14) \\
\hline $\begin{array}{l}\text { Average } \\
\text { interindividual } \\
\% C V^{b}, c \text { [range] }\end{array}$ & $\begin{array}{c}29 \pm 11 \\
{[8-46]}\end{array}$ & - & $\begin{array}{l}51 \pm 31 \\
{[12-98]}\end{array}$ & $\begin{array}{r}44 \pm 26 \\
{[4-94]}\end{array}$ & $\begin{array}{l}44 \pm 17 \\
{[28-75]}\end{array}$ & $\begin{array}{c}43 \pm 26 \\
{[3-84]}\end{array}$ & $\begin{array}{c}42 \pm 12 \\
{[17-61]}\end{array}$ & $\begin{array}{c}33 \pm 14 \\
{[8-47]}\end{array}$ & $\begin{array}{l}55 \pm 20 \\
{[21-88]}\end{array}$ & $\begin{array}{l}17 \pm 7 \\
{[6-29]}\end{array}$ \\
\hline $\begin{array}{l}\text { Average } \\
\text { intra-individual } \\
\% \mathrm{CV}^{\mathrm{d}} \text { [range] }\end{array}$ & $\begin{array}{l}22 \pm 7 \\
{[16-32]}\end{array}$ & - & $\begin{array}{l}55 \pm 27 \\
{[21-86]}\end{array}$ & $\begin{array}{l}36 \pm 8 \\
{[27-45]}\end{array}$ & $\begin{array}{l}28 \pm 14 \\
{[12-47]}\end{array}$ & $\begin{array}{l}42 \pm 9 \\
{[31-53]}\end{array}$ & $\begin{array}{l}25 \pm 14 \\
{[13-46]}\end{array}$ & $\begin{array}{c}18 \pm 12 \\
{[2-28]}\end{array}$ & $\begin{array}{l}35 \pm 15 \\
{[21-59]}\end{array}$ & $\begin{array}{l}10 \pm 7 \\
{[2-53]}\end{array}$ \\
\hline \multicolumn{11}{|l|}{ Recovery } \\
\hline 16 & 1740 (31) & - & 1717 (39) & - & $2520(52)$ & - & $1968(46)$ & - & $1220(28)$ & - \\
\hline 20 & 1744 (19) & - & $1848(31)$ & - & $1770(32)$ & - & 1945 (48) & - & 1730 (23) & - \\
\hline 24 & 1853 (19) & - & $1720(43)$ & - & $2080(41)$ & - & 2128 (33) & - & $1620(25)$ & - \\
\hline
\end{tabular}

Percent coefficient of variation (\% CV) is reported within parentheses. aLymphocyte count and reduction, $\mathrm{P}<0.05$ between groups (ANOVA); binterindividual \% CV according to group, $\mathrm{P}>0.05$ for lymphocyte count, and $\mathrm{P}<0.01$ for reduction (ANOVA); cinterindividual \% CV according to visit, $\mathrm{P}>0.05$ (general linear model for repeated measures); dintra-individual \% CV according to group, $\mathrm{P}>0.05$ for lymphocyte count and $\mathrm{P}<0.01$ for reduction (ANOVA). 

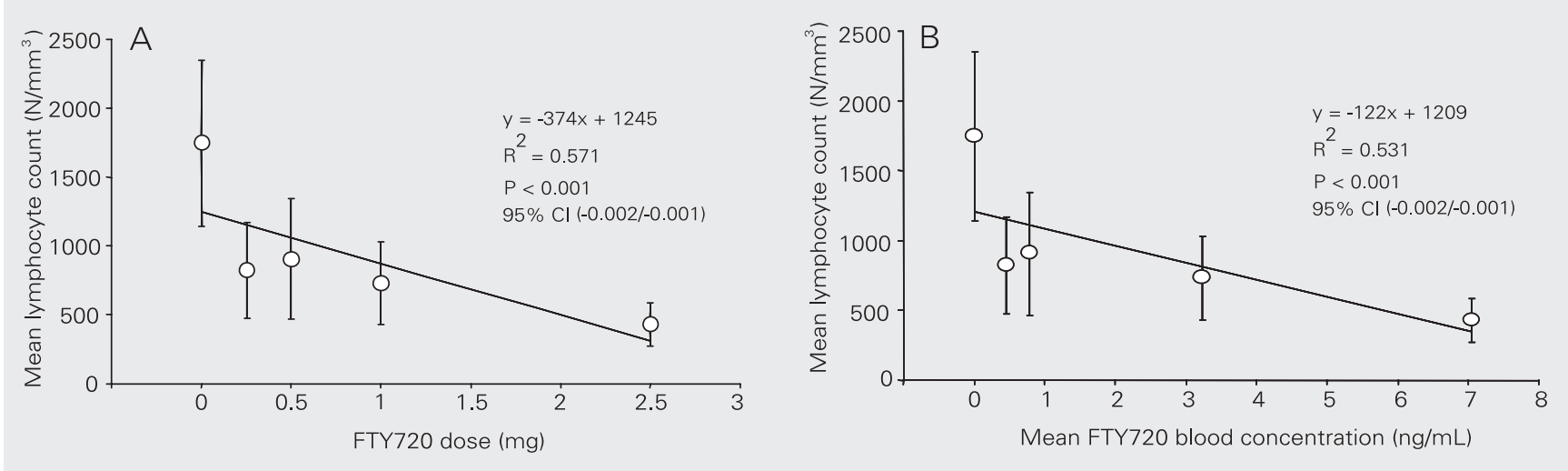

Figure 5. Linear regression analysis correlating mean FTY720-induced reduction of peripheral lymphocyte counts and drug dose (A) or concentrations (B)
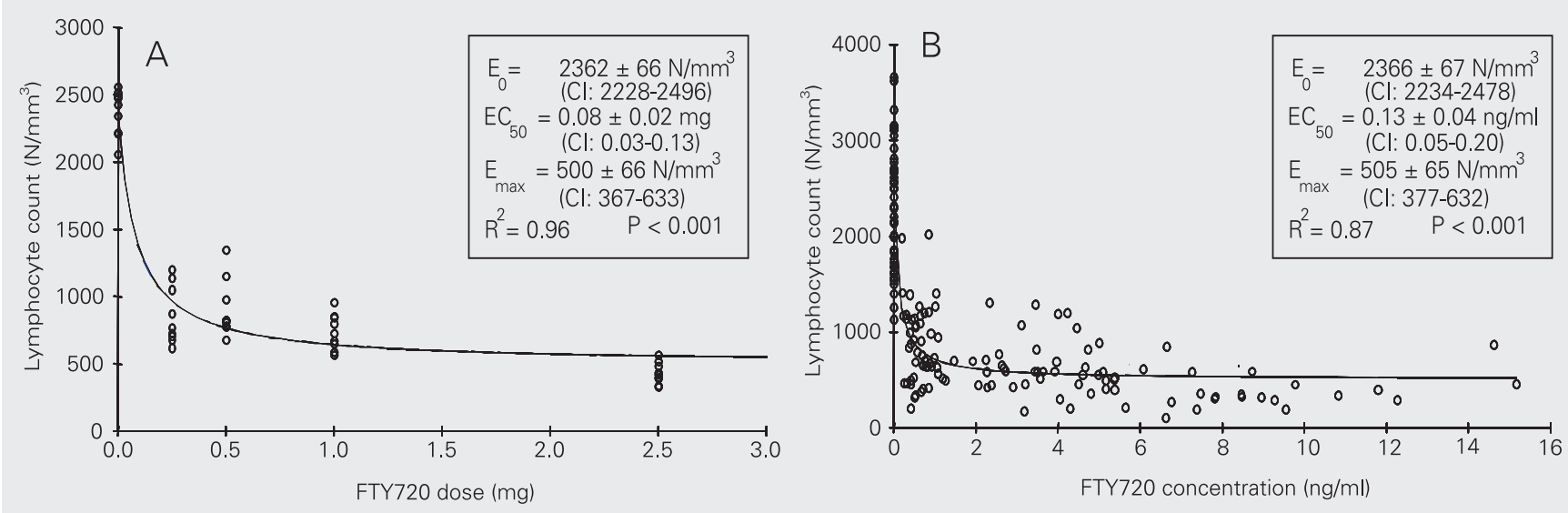

Figure 6. Pharmacokinetic and pharmacodynamic inhibitory $E_{\max }$ model correlating FTY720 dose (A) or blood concentration (B) with absolute lymphocyte counts in peripheral blood.
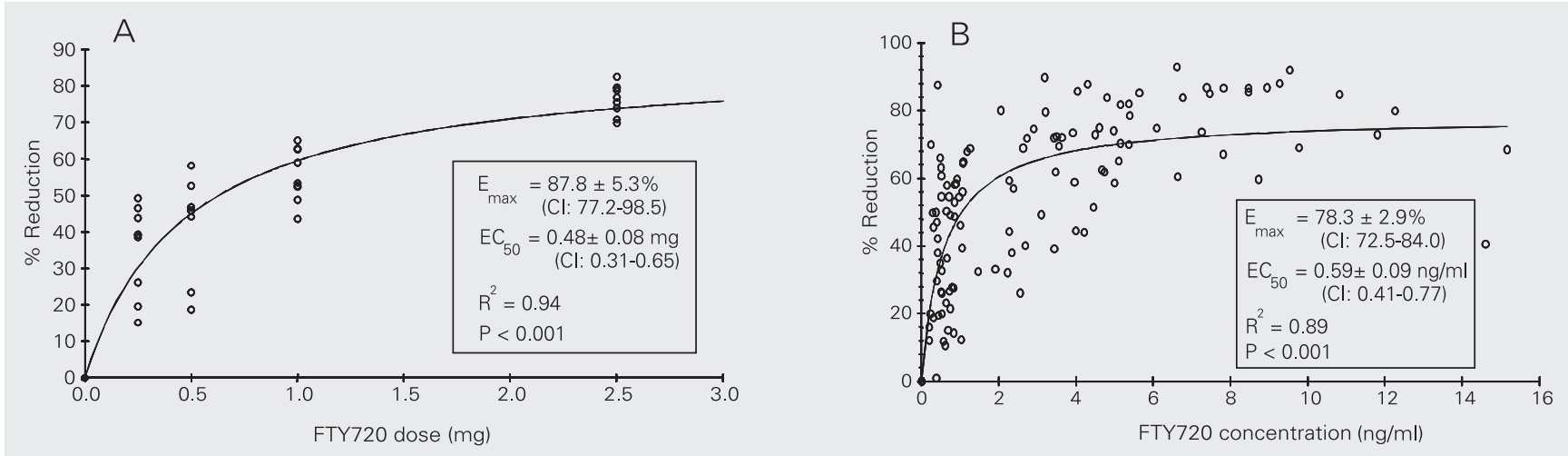

Figure 7. Pharmacokinetic and pharmacodynamic simple $E_{\max }$ model correlating FTY720 dose (A) or blood concentration (B) with percent reduction in lymphocyte counts in peripheral blood. 
of absolute lymphocyte counts did not differ according to FTY720 dose level. However, a decrease in both inter- (44-17\%) and intraindividual variability (42-10\%) ranges of percent reduction in lymphocyte counts according to FTY720 dose level was observed $(\mathrm{P}<0.01)$. There was no significant difference in interindividual $\% \mathrm{CV}$ according to study visit.

\section{PK/PD analysis}

The observed linear correlation between FTY720 dose and steady-state blood concentrations did not translate into linear correlations between dose or blood concentrations and lymphopenia. In the 10-fold dose range studied in this trial ( 0.25 to $2.5 \mathrm{mg} /$ day $)$ FTY720 pharmacodynamics was not doselinear $\left(r^{2}=0.57\right.$ and 0.53$)$, as can be observed in Figure 5A and B. Searching for a best-fit model we identified the inhibitory $\mathrm{E}_{\max }$ model as the one with best prediction of the relationship between both FTY720 dose or blood concentrations and absolute peripheral lymphocyte count. This model applies the following formula: $\mathrm{E}=\mathrm{E}_{\max }-\left(\mathrm{E}_{\max }-\mathrm{E}_{0}\right)$ $*\left[\mathrm{C} /\left(\mathrm{C}+\mathrm{EC}_{50}\right)\right]$, where $\mathrm{E}$ is the pharmacodynamic effect at a given drug dose or concentration (C), $\mathrm{E}_{\max }$ is the maximum pharmacodynamic effect attributed to the drug, $\mathrm{E}_{0}$ is the effect when drug dose or concentration is equal to zero, and $\mathrm{EC}_{50}$ is the drug dose or concentration which produces $50 \%$ of the maximum pharmacodynamic effect. The last parameter determines the drug potency. Using this model, the correlation between individual FTY720 doses or steadystate blood concentrations and absolute peripheral lymphocyte counts produced coefficients of determination of $\mathrm{r}^{2}=0.96$ and $\mathrm{r}^{2}$ $=0.87$, respectively. For FTY720 dose and concentration correlations, $\mathrm{E}_{0}$ and $\mathrm{E}_{\max }$ were very similar, with $\mathrm{EC}_{50}$ of $0.08 \mathrm{mg}$ or 0.13 $\mathrm{ng} / \mathrm{ml}$, respectively (Figure 6A,B). Conversely, the simple $\mathrm{E}_{\max }$ model was the one that best predicted the relationship between either FTY720 dose or blood concentration and percent reduction in peripheral lymphocyte count. This model applies the following formula: $\mathrm{E}=\left(\mathrm{E}_{\max } * \mathrm{C}\right) /\left(\mathrm{C}+\mathrm{EC}_{50}\right)$, where $\mathrm{E}$ is the pharmacodynamic effect at a given drug dose or concentration (C), $\mathrm{E}_{\max }$ is the maximum pharmacodynamic effect attributed to the drug, and $\mathrm{EC}_{50}$ is the drug dose or concentration which produces $50 \%$ of the maximum pharmacodynamic effect. Again, using this model, the correlation between individual FTY720 doses or steady-state blood concentrations and percent reduction in peripheral lymphocyte counts produced coefficients of determination of $\mathrm{r}^{2}=0.94$ and $\mathrm{r}^{2}$ $=0.89$, respectively. For FTY720 dose and concentration correlations, $\mathrm{E}_{\max }$ was $87.8 \%$ and $78.3 \%$, with $\mathrm{EC}_{50}$ of $0.48 \mathrm{mg}$ or $0.59 \mathrm{ng} /$ $\mathrm{ml}$, respectively (Figure 7A,B).

\section{Discussion}

FTY720 is a novel immunomodulating drug with a unique mechanism of action, which is different from the currently used immunosuppressive agents. Results from phase II clinical trials have demonstrated that the combination of FTY720 with cyclosporine produces a very low incidence of acute rejection (26). Furthermore, except for a first dose-associated mild negative chronotropic effect, FTY720 is not nephrotoxic, does not potentiate cyclosporine nephrotoxicity and lacks other specific drugrelated negative side effects (27).

In the present study, we set out to determine FTY720 PK/PD relationships in a cohort of patients undergoing a dose-finding phase II clinical trial (28). The demographic characteristics revealed a low risk transplant population since this was an exploratory study, but no significant differences were observed among the study groups (Table 1). All patients received comparable initial cyclosporine doses and underwent similar dose reductions resulting in virtually equal cyclosporine concentrations throughout the first 
24 post-transplant weeks. Moreover FTY720 did not produce any significant drug interaction with cyclosporine since blood cyclosporine concentrations were similar for all FTY720 dose groups and for the MMF group (29).

The pharmacokinetic properties of FTY720 have been described previously (30). The long terminal half-life required a loading dose to achieve steady-state concentrations more rapidly. Except for the higher dose level, whereby mean blood concentrations were still increasing after week 4 (Table 2, Figure 2), it appears that steady-state concentrations were achieved after 4 weeks of daily administration. In this cohort of patients, we observed a relatively low inter- and intra-individual variability in FTY720 trough blood concentrations, regardless of FTY720 dose or time post-transplant. Moreover, FTY720 showed dose-linear pharmacokinetics (13). Taken together, these characteristics may result in less need for dose adjustments, and fast and more predictable achievement of target concentrations when dose adjustments are needed.

As shown in Table 3, MMF did not produce lymphopenia during the study period. FTY720-induced lymphopenia began early and could be detected as early as 1 week after transplantation (Figure 4). Actually, in more detailed studies, lymphopenia was already noticed after the loading dose and paralleled the decrease in heartbeat (28). This has been attributed to the physiological mechanism of action of FTY720, which, after oral administration, is phosphorylated (FTY720P) and binds to sphingosine-1-phosphate receptors present in the plasma membrane of lymphocytes and cardiac myocytes, respectively $(31,32)$. Nadir lymphopenia is reached between 2 and 4 weeks and after drug discontinuation lymphocyte counts return to baseline values within 4 to 8 weeks depending on the magnitude of the FTY720 dose. This indicates that FTY720-induced lymphopenia is reversible and therefore safer, since it does not produce prolonged apoptosis-related lymphopenia and does not produce long-term effects like those observed using depleting monoclonal or polyclonal antibodies (33). Inter- and intra-individual variability of the peripheral absolute lymphocyte count is apparently higher than that seen for the FTY720 pharmacokinetics. Interand intra-individual variability of percent reduction of lymphocyte counts decreases with increasing FTY720 doses, perhaps due to achievement of maximal pharmacodynamic effect.

Visual observation of the relationship between FTY720 doses or blood concentration absolute lymphocyte count or percent reduction in lymphocyte count revealed a nonlinear pattern (Figure 3), supported by the low coefficient of determination $\left(\mathrm{r}^{2}\right)$ of the linear regression analysis (Figure 4). Searching for a best-fit model that would explain this relationship we found two PK/PD models. For the absolute lymphocyte count, the inhibitory $\mathrm{E}_{\max }$ model was selected since there was a decrease (inhibition) in the number of lymphocytes in peripheral blood with increasing drug dose or concentration, showing high coefficients of determinations (Figure 6). The $\mathrm{E}_{0}$ term was included to describe the effect when drug dose or concentrations were equal to zero (in this study, lymphocyte counts from patients receiving MMF). For the percent reduction in lymphocyte counts, the simple $\mathrm{E}_{\max }$ model was selected since there was an increase in percent reduction of the number of lymphocytes in peripheral blood with increasing drug dose or concentration, also showing high coefficients of determination (Figure 7). There was an evident difference in $\mathrm{EC}_{50}$ between the two PK/ PD models for both FTY720 dose $(0.08 v s$ $0.48 \mathrm{mg})$ and blood concentration $(0.13 \mathrm{vs}$ $0.59 \mathrm{ng} / \mathrm{ml}$ ). A simple explanation for this finding is the large but frequently observed variability of the peripheral lymphocyte counts when drug dose or concentrations were equal to zero $\left(\mathrm{E}_{0}\right.$ term), suggesting that the 
use of percent reduction, which naturally corrects for baseline differences, is more appropriate as a surrogate marker of the pharmacodynamic effect of FTY720 in the blood compartment.

Finally, relationships between pharmacokinetics and pharmacodynamics are useful to find ideal doses to produce the desired effect. However, it is still important to determine the relationship between the pharmacodynamic effect and efficacy and safety, which is the ultimate goal of pharmacology. In a phase II clinical trial, the 2.5-mg dose of FTY720 showed good efficacy for the prevention of acute rejection after kidney transplantation. This dose produced a reduction of about $80 \%$ in lymphocyte count, close to the maximum effect $\left(\mathrm{E}_{\max }\right)$. Since FTY720 accumulates 11 - to 19 -fold in various tissues, perhaps in the secondary lymphoid organs, which are rich in lymphocytes, and therefore in sphingosine-1-phosphate receptors, the $\mathrm{EC}_{50}$ required for drug-receptor complex internalization might be higher. Moreover, data from the same phase II clinical trial demonstrated that FTY720 at doses of $5 \mathrm{mg} /$ day, which probably produce maximum percent reduction of lymphocyte count, is still effective and safe. Recently, it has been demonstrated that the active form of FTY720 is the phosphorylated form (FTY720-P) and studies are needed to assess the correlation between FTY720-P and the degree of lymphopenia. Of course, the lacks of similar dose-response curves for toxicity impair the establishment of a therapeutic window.

In conclusion, the PK/PD relationship of FTY720 follows an $\mathrm{E}_{\max }$ model. Using peripheral lymphopenia as an FTY720 pharmacodynamic surrogate marker, high pharmacodynamic effects (percent reduction in peripheral lymphocytes) are required to achieve best efficacy, similar to what is seen in various physiological enzymatic systems. Further prospective studies are required to test the clinical applicability of this strategy.

\section{Acknowledgments}

We are indebted to Dr. John Kovarik, Novartis PharmD AG, Basel, Switzerland, for a critical review of the manuscript.
1. Adachi K, Kohara T, Nakao N, Arita M, Chiba K, Mishina T, Sasaki S \& Fujita $T$ (1995). Design, synthesis, and structure-activity relationships of 2-substituted-2-amino-1,3-propanediols: Discovery of a novel immunosuppressant, FTY720. Biomedicinal Chemistry Letters, 5: 847-852.

2. Kiuchi M, Adachi K, Kohara T, Teshima K, Masubuchi Y, Mishina T \& Fujita $T$ (1998). Synthesis and biological evaluation of 2,2disubstituted 2-aminoethanols: analogues of FTY720. Bioorganic and Medicinal Chemistry Letters, 8: 101-106.

3. Kiuchi M, Adachi K, Kohara T et al. (2000). Synthesis and immunosuppressive activity of 2-substituted 2-aminopropane-1, 3-diols and 2-aminoethanols. Journal of Medicinal Chemistry, 43: 29462961.

4. Fujita $T$, Inoue $K$, Yamamoto $S$, Ikumoto $T$, Sasaki $S$, Toyama $R$, Yoneta M, Chiba K, Hoshino Y \& Okumoto T (1994). Fungal metabolites. Part 11. A potent immunosuppressive activity found in Iscaria sinclairii metabolite. Journal of Antibiotics, 47: 208-215.

5. Troncoso P \& Kahan BD (1998). Preclinical evaluation of a new immunosuppressive agent, FTY720. Clinical Biochemistry, 31: 369-373.
6. Furukawa H, Suzuki T, Jin MB et al. (2000). Prolongation of canine liver allograft survival by a novel immunosuppressant, FTY720: effect of monotherapy and combined treatment with conventional drugs. Transplantation, 69: 235-241.

7. Quesniaux V, Menninger K, Audet M, Gaschen L \& Schuurman HJ (2001). FTY-720 is efficacious in monkey kidney transplantation. Transplantation Proceedings, 33: 2374-2375.

8. Troncoso P, Stepkowski SM, Wang ME, Qu X, Chueh SC, Clark J \& Kahan BD (1999). Prophylaxis of acute renal allograft rejection using FTY720 in combination with subtherapeutic doses of cyclosporine. Transplantation, 67: 145-151.

9. Nikolova Z, Hof A, Baumlin Y \& Hof R (2001). Combined FTY720/ cyclosporine treatment promotes graft survival and lowers the peripheral lymphocyte count in a murine cardiac allotransplantation model. Transplantation, 72: 168-171.

10. Xu M, Pirenne J, Antoniou EA, Afford SC, D'Silva M \& McMaster P (1998). Effect of peritransplant FTY720 alone or in combination with posttransplant FK 506 in a rat model of cardiac allotransplantation. Transplant International, 11: 288-294.

11. Tamura A, Li XK, Funeshima N, Enosawa S, Amemiya H, Kitajima M 
\& Suzuki S (2000). Immunosuppressive therapy using FTY720 combined with tacrolimus in rat liver transplantation. Surgery, 127: 47-54.

12. Wang ME, Tejpal N, Qu X, Yu J, Okamoto M, Stepkowski SM \& Kahan BD (1998). Immunosuppressive effects of FTY720 alone or in combination with cyclosporine and/or sirolimus. Transplantation, 65: 899-905

13. Quesniaux VF, Menninger K, Kunkler A, Vedrine C, Bernhard M, Hedinger R, Kraus G \& Schuurman HJ (2000). The novel immunosuppressant FTY720 induces peripheral lymphodepletion of both $\mathrm{T}$ - and B-cells in cynomolgus monkeys when given alone, with cyclosporine neoral or with RAD. Transplant Immunology, 8: 177187.

14. Nikolova Z, Hof A, Baumlin Y \& Hof RP (2001). Efficacy of SDZ RAD compared with CsA monotherapy and combined RAD/FTY720 treatment in a murine cardiac allotransplantation model. Transplant Immunology, 9: 43-49.

15. Brinkmann V, Pinschewer DD, Feng L \& Chen S (2001). FTY720: altered lymphocyte traffic results in allograft protection. Transplantation, 72: 764-769.

16. Rosen H, Sanna G \& Cheistopher A (2003). Egress: a receptorregulated step in lymphocyte trafficking. Immunological Reviews, 195: 160-177.

17. Pinschewer DD, Ochsenbein AF, Odermatt B, Brinkmann V, Hengartner H \& Zinkernagel RM (2000). FTY720 immunosuppression impairs effector $\mathrm{T}$ cell peripheral homing without affecting induction, expansion, and memory. Journal of Immunology, 164: 5761-5770

18. Brinkmann V, Chen S, Feng L, Pinschewer D, Nikolova Z \& Hof R (2001). FTY720 alters lymphocyte homing and protects allografts without inducing general immunosuppression. Transplantation Proceedings, 33: 530-531.

19. Budde K, Schmouder RL, Brunkhorst R, Nashan B, Lucker PW, Mayer T, Choudhury S, Skerjanec A, Kraus G \& Neumayer $\mathrm{HH}$ (2003). Pharmacodynamics of single doses of the novel immunosuppressant FTY720 in stable renal transplant patients. American Journal of Transplantation, 3: 846-854.

20. Pyne S \& Pyne NJ (2000). Sphingosine 1-phosphate signaling in mammalian cells. Biochemical Journal, 349: 385-402.

21. Brinkmann V, Davis MD, Heise CE et al. (2002). The immune modulator, FTY720, targets sphingosine 1-phosphate receptors. Journal of Biological Chemistry, 277: 21453-21457.

22. Matloubian M, Lo CG, Cinamon G, Lesneski MJ, Xu Y, Brinkmann V, Allende ML, Proia RL \& Cyster JG (2004). Lymphocyte egress from thymus and peripheral lymphoid organs is dependent on S1P receptor 1. Nature, 427: 355-360.

23. Mandala S, Hajdu R, Bergstrom J et al. (2002). Alteration of lymphocyte trafficking by sphingosine-1-phosphate receptor agonists. Science, 296: 346-349.

24. Chen S, Bacon KB, Garcia G, Liao R, Pan ZK, Sullivan SK, Nakano H, Matsuzawa A, Brinkmann V \& Feng L (2001). FTY720, a novel transplantation drug, modulates lymphocyte migratory responses to chemokines. Transplantation Proceedings, 33: 3057-3063.

25. Allison AC \& Eugui EM (2000). Mycophenolate mofetil and its mechanisms of action. Immunopharmacology, 47: 85-118.

26. Kovarik JM, Tedesco-Silva H, Schmouder R, Pellet P \& Fornairon S (2003). Associations between FTY720 blood levels vs lymphopenia and acute rejection episodes in de novo kidney transplantation. American Journal of Transplantation, 3 (Suppl V): 308 (Abstract).

27. Kahan BD, Karlix JL, Ferguson RM, Leichtman AB, Mulgaonkar S, Gonwa TA, Skerjanec A, Schmouder RL \& Chodoff L (2003). Pharmacodynamics, pharmacokinetics, and safety of multiple doses of FTY720 in stable renal transplant patients: a multicenter, randomized, placebo-controlled, phase I study. Transplantation, 76: 1079-1084.

28. Tedesco H, Kahan B \& Mourad G (2001). FTY720 Combined with Neoral ${ }^{T M}$ and Corticosteroids is Effective and Safe in Prevention of Acute Rejection in Renal Allograft Recipients (interim data). Transplant2001, Chicago, IL, USA, 429.

29. Nikolova Z, Hof A, Baumlin $Y$ \& Hof RP (2000). The periphera lymphocyte count predicts graft survival in DA to Lewis heterotopic heart transplantation treated with FTY720 and SDZ RAD. Transplant Immunology, 8: 115-124

30. Tedesco-Silva Jr H, Felipe CR \& Medina-Pestana JO (2002). FTY720. Current Opinion in Organ Transplantation, 7: 342-352.

31. Liliom K, Sun G, Bunemann M et al. (2001). Sphingosylphosphocholine is a naturally occurring lipid mediator in blood plasma: a possible role in regulating cardiac function via sphingolipid receptors. Biochemical Journal, 355: 189-197.

32. Mazurais D, Robert P, Gout B, Berrebi-Bertrand I, Laville MP \& Calmels T (2002). Cell type-specific localization of human cardiac S1P receptors. Journal of Histochemistry and Cytochemistry, 50: 661-669.

33. Brennan DC, Flavin K, Lowell JA et al. (1999). A randomized, double-blinded comparison of Thymoglobulin versus Atgam for induction immunosuppressive therapy in adult renal transplant recipients. Transplantation, 67: 1011-1018. 